\title{
LE MYTHE ESCHATOLOGIQUE CHEZ PLATON Introduction
}

L'influence de la pensée platonicienne sur notre conscience dans le domaine de l'eschatologie est telle que souvent nous ne nous rendons pas compte à quel point elle est profondément enracinée dans notre culture et notre pensée. La connaissance des sources est en ce cas très importante pour comprendre qu'aujourd'hui il est pour nous très difficile de penser à l'eschatologie sans ces images que nous a laissées Platon. Par eschatologie nous comprenons ici ce qui concerne le destin de l'homme après la mort: jugement, destinée de l'homme, réincarnation et aussi - avant sa vie - sa préexistence.

Les sources principales pour la connaissance du mythe eschatologique chez Platon sont assez nombreuses. Platon nous donne des images du destin des âmes avec vision cosmologique du monde dans les dialogues suivants: 1. Phédon - en particulier: 81-82, 106-108, 111-114; 2. Phèdre - 237, 245-250 (préexistence), 250-257 (anthropologie), 3. Gorgias - 523-527 (le mythe des enfers), 4. La République - 614-621 (le mythe d'Er), 5. Les Lois - 870 sqq. (théorie de l'expiation), 903-904 (le but de la vie humaine et la destinée de l'homme) ${ }^{1}$. Nous avons indiqué ici seulement les fragments de base. Dans le texte nous en indiqueront aussi d'autres, plus petits.

\section{LE SCHÉMA ANTHROPOLOGIQUE DANS LES ÉCRITS DE PLATON}

C'est la première et la plus valable constatation d' anthropologie platoni-

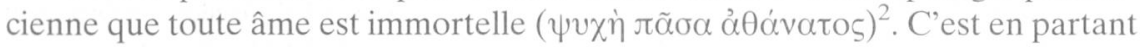

1 Tous les passages des dialogues sont cités en français selon l'Edition des Belles Lettres de Paris: Phédon, texte établi et traduit par L. Robin: Platon, Oeuvres complètes, III/1, Paris 1923; Phèdre, texte établi et traduit par L. Robin: Platon, Oeuvres complètes, IV, Paris 1933; Gorgias, texte établi et traduit par A. Croiset avec la collaboration de Louis Bodin: Platon, Oeuvres complètes, III/2, Paris 1923; La République, texte établi et traduit par E. Chambry: Platon, Oeuvres complètes, VI-VII, Paris 1932; Les Lois, texte établi et traduit par E. des Places: Platon, Oeuvres complètes, XI-XII, Paris 1951-1956.

2 Cfr. Phaedrus $245 \mathrm{c}$. 
de ce principe que l'on arrive à la conclusion que ce qui se meut soi-même (l'âme) doit être nécessairement à la fois inengendré et immortel ${ }^{3}$. L'âme est mue par elle-même et voilà ce qui décide de l'essence de l'âme. En ce mouvement de l'âme on doit distinguer deux tendances contraires. L'une: suivre le corps, l'autre: suivre la raison qui distingue le bien ${ }^{4}$. Platon compare l'âme à un attelage ailé. Cet attelage est tiré par deux chevaux ${ }^{5}$. L'un est noir et il veut suivre le corps, l'autre est blanc et obéissant à la raison. Ils sont comme deux êtres qui habitent le corps. Platon dit:

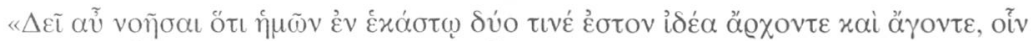

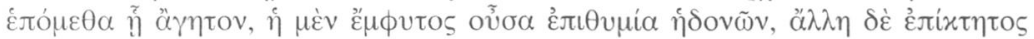

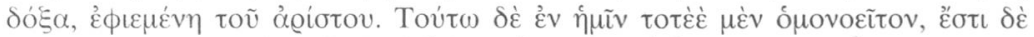

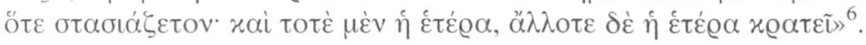

L'âme par rapport au corps est celle qui commande et le corps est celui qui obéit ${ }^{7}$.

La mort selon Platon est la séparation de l'âme et du corps ${ }^{8}$. L'âme après la mort de l'homme garde «tous ses traits naturels et toutes les modifications qu'elle a subies par suite des manières de vivre auxquelles l'homme l'a pliée en chaque circonstance», elle garde sa nature propre ${ }^{9}$. Ces constatations ont une grande importance pour considérer l'idée du jugement, de la peine et de la récompense après la mort en tenant compte du libre arbitre de l'homme et donc de sa responsabilité.

\section{PRÉEXISTENCE ET BUT DE LA VIE HUMAINE}

Dieu, qui créa l'univers, créa de même toutes les âmes ${ }^{10}$ qui sont destinées

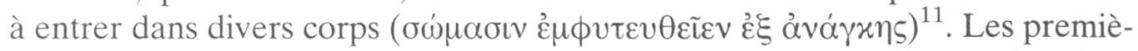
res naissances sont égales pour toutes les âmes. Au début, elles ont la même chance $^{12}$. Avant sa naissance, l'âme prend sa part à la révolution de l'orbe

${ }^{3}$ Cfr. ibidem 246 a.

${ }^{4}$ Cfr. ibidem $237 \mathrm{~d}$.

5 Cfr. ibidem 246 a-e.

${ }^{6}$ Ibidem 237d-e: «Il faut réfléchir, qu'en chacun de nous il existe deux formes de principes et de motifs d'action, que nous suivons où ils peuvent bien nous mener: l'un, qui est inné, est le désir des plaisirs; l'autre, qui est une façon de voir acquise, aspire au meilleur. Or ces deux tendances sont en nous parfois concordantes, mais il arrive aussi qu'elles soient en lutte, et c'est parfois celleci qui domine, mais d'autres fois c'est celle-là».

7 Cfr. Leges 896 c.

8 Cfr. Gorgias 524 b; Phaedo 64 c.

${ }^{9}$ Cfr. Gorgias 524 d.

${ }^{10}$ Cfr. Timaios 41 d-e.

11 Ibidem 42 a ; cfr. Phaedrus, 245 c-246 a - la preuve de l'éternité de l'âme - et 248 c-249 d.

12 Cfr. Timaios 41 e. 
céleste qui s'accomplit tous les $10.000 \mathrm{ans}^{13}$. Nous voyons ici le cortège qui court le monde céleste pour contempler les réalités véritables. En ce cortège marchent en tête les âmes des dieux et en dernier les âmes des hommes qui les suivent. Chaque âme fait de son mieux pour suivre les dieux car ils guident à l'endroit d'où les âmes peuvent voir le monde céleste des réalités véritables ${ }^{14}$. Au milieu de ce monde est situé l'être réel. C'est la raison, le guide de l'âme, qui seule peut voir et contempler cet être réel. Les âmes des hommes ne peuvent pas voir exactement et parfaitement ce monde des réalités véritables qui entourent l'être réel. Les chevaux de l'attelage de l'âme font toujours obstacle. Parmi les âmes l'une «élève vers le lieu qui est en dehors du ciel la tête de son cocher; entraînée dans la révolution circulaire, elle est à grand peine capable, dans embarras que ses chevaux lui causent, de porter les yeux sur les réalités. L'autre tantôt lève, tantôt enfonce sa tête et, ne maîtrisant pas ses chevaux, elle voit les unes et non les autres» ${ }^{15}$. Bien que l'attelage de l'âme soit ailé pour élever vers le haut ce qui est pesant, elle peut perdre une propriété de sa nature qui peut être détruite par ce qui est le contraire du beau, du savant et du bon ${ }^{16}$. Elle doit donc à toute force suivre les dieux car selon le décret

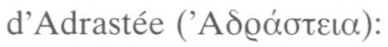

«toute âme qui, en faisant partie du cortège d'un dieu, a eu quelque vision des

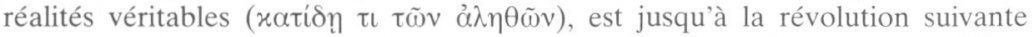
exempte d'épreuve, et, si toujours elle est capable de réaliser cette condition, pour toujours elle est exempte de dommage» ${ }^{17}$.

Mais, cette âme qui s'enfonce et tombe ou ne s'élève pas, doit être punie. Elle entre dans un corps d'animal. Au contraire, celle qui a vu le plus, devient l'âme de l'homme qui est un philosophe, qui se consacre au Beau, aux Muses, à l'amour ${ }^{18}$. En tous les cas la loi d'Adrastée "concerne la destinée finale des âmes, par rapport à ce qui sera leur existence dans la vie terrestre et après» ${ }^{19}$. L'âme qui n'a point vu les réalités véritables ne peut entrer que dans un organisme d'animal; celle qui a vu le plus entre dans un corps d'homme. Quelques-unes, par l'effet de quelque disgrâce pleines d'oubli et de perversion, s'alourdissent ${ }^{20}$.

${ }^{13}$ Cfr. Phaedrus $248 \mathrm{~d}$

${ }^{14}$ Cfr. ibidem 248 c.

15 Ibidem 248 a.

16 Cfr. L. Robin, Notice, in: Platon, Oeuvres complètes. Phèdre, texte établi et traduit par L. Robin, IV/3, Paris 1933, p. LXXXII.

${ }_{17}$ Phaedrus $248 \mathrm{c}$.

18 Cfr. ibidem 248 c-d.

19 Cfr. Robin, Notice, p. LXXXVII-LXXXVIII.

${ }^{20}$ Cfr. Phaedrus 248 c; Phaedo 81 c-e. 
Quelle est alors leur destinée après leur décadence? Il y a deux sortes d'âmes qui ont déchu. Les unes, celles qui ont vu quelque chose, elles ont eu une certaine part à la contemplation du monde céleste, des réalités vraies et les autres qui en ont été privées ${ }^{21}$. Léon Robin remarque qu'il y a dans Phèdre une double eschatologie par rapport aux âmes qui ont déchu ${ }^{22}$. Une destinée «dépend de l'antérieur à la vie terrestre» et l'autre est la conséquence de cette vie ${ }^{23}$

Léon Robin ${ }^{24}$ distingue ici alors deux eschatologies. La première concerne une hiérarchisation des espèces d'hommes et de leurs occupations d'après les qualités des âmes, proportionnellement au degré de contemplation des réalités vraies. Les âmes qui ont bien suivi les dieux, pour bien voir ces réalités, sont exemptes de dommage. L'objet de la seconde eschatologie est la question que deviennent les âmes après la première mort. Il y a deux questions: 1. Leur sort dépend de leurs vies dans leurs corps par rapport à la justice et au bien ${ }^{25}$. 2. Tous les 1000 ans l'âme commence sa nouvelle vie terrestre, mais après 10000 ans elle revient, de nouveau ailée, pour prendre sa place dans le cortège céleste. Nous ne savons pas si toutes les âmes peuvent de nouveau prendre part dans ce choeur divin, car dans l'eschatologie du Phédon le retour «est pour toujours refusé aux grands coupables» ${ }^{26}$, tandis que dans la République peut-être il s'agit de possibilité de recommencer la vie après mille ans ${ }^{27}$. Cela veut dire qu'il y a la possibilité pour l'âme de reprendre la place qu'elle avait au debut.

Il nous reste la question: quel est selon Platon le but de la vie humaine? Il semble qu'on doit considérer deux aspects de cette vie: l'un subjectif, personnel et l'autre universel. Dans le sens subjectif c'est le bonheur de l'âme après la mort, mais en gardant le souvenir que l'homme ne vit pas seulement pour luimême; dans le sens objectif c'est l'harmonie et la félicité de l'univers. Dans les Lois, Platon dit:

«Simple unité que tu es dans ce nombre, ô chétif ergoteur, ta part de rôle toujours tend et vise à l'ensemble, si minime qu'elle soit, et tu n'as pas conscience, dans tout ce drame, que rien ne se fait sinon pour cette fin, d'assurer, à la vie de l'univers, permanence et félicité, et que rien ne se fait pour toi, mais toi pour l'ensemble» ${ }^{28}$.

${ }^{21}$ Cfr. Robin, Notice, p. LXXXVI.

22 Elle a d'autres analogues en Timée : 41 d-42 d, 90 a-c, 91 d-92 c.

${ }^{23}$ Cfr. Robin, Notice, p. LXXXVII.

${ }^{24}$ Cfr. ibidem.

25 Cfr. ibidem, p. XC; voir aussi Leges $903 \mathrm{c}$.

26 Phaedo 113 e: «Il en est d'autres dont l'état aura été reconnu sans remède à cause de la grandeur de leurs fautes [...], le lot qui conviennt à ceux-là, c'est d'être lancés dans le Tartre, d'où plus jamais ils ne sortent».

27 Cfr. Respublica 615 a-c.

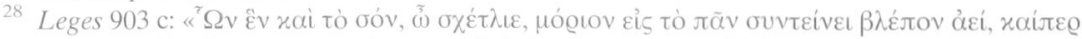

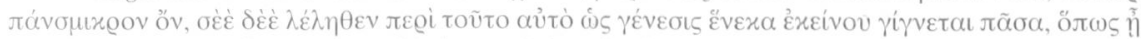

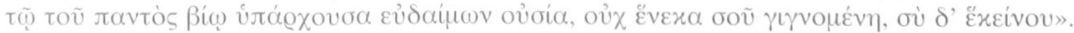




\section{LA DESTINÉE DE L'HOMME APRÈS LA VIE TERRESTRE}

\section{A. Le jugement}

1. Les juges et le lieu du jugement. Le jugement est la première chose que subit une âme après la mort de l'homme. Dans le Phédon Platon dit que le jugement s'accomplit avant la descente de l'âme chez Hadès. Ensuite elle est conduite par son génie, qui avait soin d'elle pendant la vie de l'homme, à sa destination chez Hadès ${ }^{29}$. Zeus y a institué comme juges trois de ses fils : Minos et Rhadamante de l'Asie et Éaque de l'Europe. Rhadamante est désigné pour juger les hommes qui viennent d'Asie et Éaque ceux qui viennent d'Europe. $\mathrm{Au}$ cas où leurs jugements paraîtraient douteux, c'est Minos qui donne la sentence définitive ${ }^{30}$.

Par nécessité le juge comme l'âme qui vient, est nu et mort pour bien faire le jugement: sans influence de la richesse, de la dignité etc., sans toutes les choses que l'âme a laissées sur la terre ${ }^{31}$. L'âme qui arrive au lieu du jugement se met devant Rhadamante. Il la considère sans savoir à qui elle appartient ${ }^{32}$. Il ne sait de l'âme qui se place devant lui, rien qu'une seule chose : si elle est mauvaise ou non ${ }^{33}$.

Les juges donnent leurs sentences dans une prairie, au carrefour, d'où mènent les deux routes, aux Iles Fortunées et au Tartare ${ }^{34}$. Le lieu du jugement est décrit plus précisément dans la République (le mythe d'Er). C'est un endroit merveilleux où il y a deux ouvertures dans la terre et en face, deux ouvertures dans le ciel. Entre ces ouvertures sont assis les juges ${ }^{35}$ et donnent leurs sentences.

2. Le jugement, la sentence et le lieu de la destination. La tâche des juges

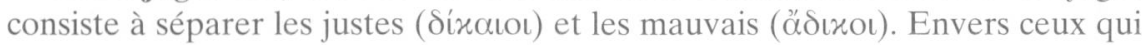
ont bien passé leur vie on a prévu les récompenses ainsi que les châtiments pour ceux qui ont été injustes. La grandeur de la récompense et de la peine dépend de la vie, de la sorte d'occupation, mais avant tout de la relation de l'homme dans sa vie au bien et à la vérité. Pour apprécier l'âme qui vient subir son jugement, les juges prennent en considération les critères négatifs, mais

${ }^{29}$ Cfr. Phaedo 107 d.

${ }^{30}$ Cfr. Gorgias 524 a ; dans l'Apologie Platon dit aussi sur les autres juges. Il ajoute là Triptolème et d'autres justes. Il dépend dans ces images de l'orphism ; voir aussi C. Clemen, Das Leben nach dem Tode im Glauben der Menschheit, Leipzig 1920, 85.

31 Cfr. Gorgias 523 b.

${ }^{32}$ Cfr. ibidem 524 d.

${ }^{33}$ Cfr. ibidem 526 b.

${ }^{34}$ Cfr. ibidem 524 a; Phaedo 107 d.

35 Cfr. Respublica 614 b-c. 
avant tout le triple critère positif: la beauté ( $x \dot{\alpha} \lambda \lambda \mathrm{s})$, la proportion

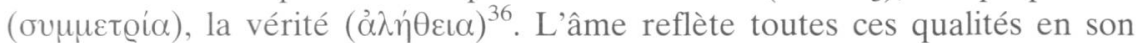
air. Dépouillée de son corps elle garde tous les traits qu'elle a subis en ellemême pendant sa vie par sa manière de vivre, par le mode de se conduire en chaque circonstance de la vie ${ }^{37}$. Celui qui meurt après une vie entièrement juste et sainte va dans les Iles des Bienheureux pour y séjourner dans la félicité, sans aucun mal. L'âme injuste et impie doit aller au lieu de la peine, au $\operatorname{Tartare}^{38}$.

Le but de la peine est double: elle doit améliorer le coupable et servir d'exemple pour les autres. Le deuxième cas est celui où le mal de l'âme est incurable: Personne ne peut guérir l'âme qui ne voulait pas guérir (pendant sa vie): elle n'a aucun avantage de sa peine. En ce cas la souffrance n'a pas de mesure et ne finit jamais ${ }^{39}$. Dans le premier cas la souffrance est un remède pour l'âme. Nous avons dit que Rhadamante ne sait rien de l'homme qui se place devant lui sauf une seule chose: s'il est mauvais. Quand il s'en assurera, il l'enverra au Tartare en lui donnant le signe (s'il est guérissable ou non ${ }^{40}$ ).

Le lieu de la destination dépend de la sentence que rendent les juges. Au cas où ils constateraient que le coupable n'est pas guérissable, il descendrait dans les plus profonds endroits du Tartare, d'où il ne pourrait jamais sortir. Si l'âme appartient à un homme qui n'a pas bien vécu, mais son malheur est guérissable, elle descend aussi au Tartare, mais après expiation elle peut en sortir. Ceux qui ont bien vécu leur vie, avant tout les philosophes, vont aux Iles des Bienheureux ${ }^{41}$.

Il y a donc deux sens contraires dans lesquels vont les âmes. La description de la République nous montre aussi deux routes. L'une, qui est à droite, mène sous la terre ${ }^{42}$. Les juges, qui siègent entre les ouvertures dans la terre et dans le ciel, commandent aux justes d'aller à droite dans le ciel leur ayant attaché par devant une plaque avec la description de leur jugement, et aux criminels d'aller à gauche sous la terre en leur suspendant par derrière un écriteau qui contient tous leurs délits ${ }^{43}$.

${ }^{36}$ Cfr. Philebus 65 a. Comme les vertus principals de l'âme Platon détaille dans le Phédon

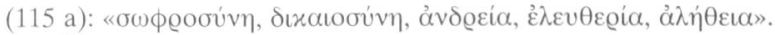

37 Cfr. Gorgias 524 b.

${ }^{38}$ Cfr. ibidem 523 a-b.

39 Cfr. ibidem 525 b.

${ }^{40} \mathrm{Cfr}$. ibidem 526 b.

41 Cfr, ibidem $526 \mathrm{c}$.

${ }^{42}$ Cfr. ibidem 614 b.

${ }^{43}$ Cfr. ibidem 614 c-d. 


\section{B. Le Tartare et le ciel. La règle de la rétribution. La grandeur et la durée de la peine}

L'endroit où se trouve le Tartare, est décrit dans le mythe cosmogonique du Phédon ${ }^{44}$. C'est un abîme, un gouffre le plus grand de tout, qui s'étend d'un bord de la terre jusqu'à l'autre. Là coulent toutes les rivières. Toutes les rivières sortent de là. Parmi ces rivières il y a quatres fleuves, dont le plus grand est l'Océan qui fait le tour de la terre. Dans le sens contraire circule Achéron qui se jette dans le lac Achérousias. Entre ces deux fleuves coule vers les endroits plus profonds du Tartare le troisième: fleuve des flammes - Pyriphlégéton. Vis-à-vis est situé le fleuve du Styxe qui se jette, comme l'Acheront, dans le lac Achérousias. Les poètes le nomment Cocyte ${ }^{45}$.

Dans le Tartare descendent deux sortes d'hommes. Ceux qui sont les plus coupables, mais guérissables, qui descendent au Tartare avec la possibilité d'en sortir. Un an après, une onde les prend et les porte au courant du Cocyte ou Pyriphlégéton dans le lac Acherousias. Ils appellent alors ceux à qui ils ont fait tort. Ils leur demandent pardon et les prient de leur permettre de sortir au bord du lac. Si leurs prières sont exaucées, leur souffrance est finie, sinon l'onde les porte de nouveau au Tartare. Leur souffrance ne finit que s'ils obtiennent la grâce de ceux qu'ils ont lésés ${ }^{46}$. Selon le mythe de la République les âmes font leur cours sous la terre pendant $1000 \mathrm{ans}^{47}$. Elles souffrent dix fois pour chaque crime parce que chaque expiation dure 100 ans, ce qui est la longueur d'une vie humaine. La proportion des récompenses est la même ${ }^{48}$.

Les Orphiques enseignaient que la Justice punit absolument, exige le redressement et fait que la faute chaque fois entraîne une expiation ${ }^{49}$. Dans les Lois Platon donne encore une autre conception de l'expiation. Après le séjour chez Hadès «une fois revenus ici (sur la terre - J.L.), leurs auteurs (des crimes J.L.) en paient nécessairement la peine naturelle en subissant à leur tour ce que eux mêmes ont fait et ne terminent leur vie actuelle qu'en souffrant de la main d'autrui les mêmes violences» ${ }^{50}$. Chacun doit inévitablement subir les mêmes violences qu'il avait infligées ${ }^{51}$.

${ }^{44}$ Cfr. Phaedo 108 b-110 e.

${ }^{45}$ Ce mythe du Phédon a un caractère un peu géographique.

${ }^{46}$ Cfr. Phaedo 114 a-b.

47 Cfr. Vergilius, Aeneis VI 748-751: «Has omnis, ubi mille rotam volvere per annos, / Lethaeum ad fluvium deus evocat agmine magno, / scilicet immemores supera ut convexa revisant / rursus, et incipiant in corpora velle reverti».

${ }^{48}$ Cfr. Respublica $615-616$.

49 Cfr. M. Maykowska, in: Platon, Prawa, thum. M. Maykowska, Warszawa 1960, p. 612, note 17 .

50 Cfr. Leges 870 d-e.

51 Cfr. ibidem 872 e. 
La théorie expiatoire de la peine dans les Lois concorde avec celle qui se trouve dans le Gorgias. ${ }^{52}$ D'autre part Protagoras, ce sophiste (dans le dialogue sous le même titre) prononce les mots suivants avec lesquels Platon semble être d'accord: «Personne, en effet, en punissant un coupable, n'a en vue ni ne prend pour mobile le fait même de la faute commise, à moins de s'abandonner comme une bête féroce à une vengeance dénuée de raison: celui qui a souci du passé car ce qui est fait est fait (soul. J.L.) - mais en prévision de l'avenir, afin que ni le coupable ni les témoins de sa punition ne soient tentés de recommencer. Penser aussi, que la vertu peut s'enseigner, s'il est vrai que le châtiment a pour fin l'intimidation ${ }^{53}$. Il y a donc des cas où le coupable ne peut pas être amélioré, mais sa peine sert comme exemple pour les autres.

\section{LA RÉINCARNATION. LE RETOUR SUR LA TERRE}

Ceux qui ont fini leur séjour au sein de la terre ou au ciel, reviennent sur la terre. Les âmes qui montent du Tartare sont exténuées et poussiéreuses, celles qui descendent du ciel toutes pures. Elles semblent venir d'un long voyage. Elles s'assemblent dans la prairie pour y camper. Celles qui se connaissent se saluent et parlent de ce qui se passait sous la terre et au $\operatorname{ciel}^{54}$. Les âmes restent dans la prairie sept jours et ensuite elles vont vers une grande lumière, d'où elles voient les rangs ( $\sigma \phi o ́ v \delta v \lambda o$ - une chose qui tourne ${ }^{55}$ ) du ciel,

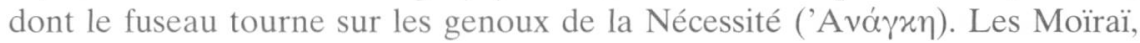
filles de la Nécessité, chantent d'accord avec les sirènes qui se trouvent au dernier rang du ciel. Lachésis chante le passé, Clotho le présent, Atropos l'avenir. Les âmes qui arrivent se présentent à Lachésis. Un hiérophante de Lachésis les range en ordre, il prend des lots sur les genoux de Lachésis et il les jette devant les âmes. Chaque lot a son numéro. Celle qui aura pris le premier, choisira la première. Elle doit nécessairement subir la vie qu'elle a choisie. Dans ce choix elle est entièrement libre et elle prend la responsabilité de ce qu'elle a choisi. La décision qu'en ce moment fait chaque homme, a une très grande valeur pour la vie à l'avenir. On doit ainsi toujours d'apprendre à être capable de choisir entre une vie bonne et une vie mauvaise. Le meilleur choix c'est de prendre après la mort une vie bonne que nous pouvons rendre meilleure ${ }^{56}$.

52 Elle concorde aussi avec celle du Phédon; cfr. 80 e-84 b ; 113 d-115 a.

53 Protagoras 324 a-b.

54 Cfr. Respublica 614 d-e.

55 Cfr. ibidem $616 \mathrm{c}$.

56 En conséquence des divers choix les âmes des animaux passent «à la condition d'hommes ou à celle d'autres animaux, les animaux injustes dans les espèces sauvages, les justes dans les espèces paisibles » etc. Il se fait des mélanges de toutes sortes, cfr. Respublica 620 d. 
En faisant le choix chez Hadès on doit se garder de se laisser éblouir par la richesse et le désir de la puissance. Parmi les diverses conditions on doit choisir toujours celle qui est moyenne. C'est le moyen par lequel l'homme peut devenir plus heureux. A l'ordinaire les hommes chez Hadès choisissent les plus mauvaises sortes en pensant qu'ils peuvent devenir heureux avec la richesse et la puissance des tyrans. Mais le meilleur choix peut seulement être fait par celui qui s'attache à la philosophie ${ }^{57}$.

Après avoir fait leur choix, les âmes vont vers Lachésis. Elle donne à chacune un génie qu'elle a choisi pour chaque âme. Le génie mène l'âme vers Clotho pour ratifier le choix que l'âme a fait. Il touche le fuseau et mène «à la trame d'Atropos pour rendre irrévocable ce qui avait été filé par Clotho». L'âme vient ensuite au pied du trône de la Nécessité. Enfin toutes les âmes passent dans la pleine du Léthé par une grande et horrible chaleur. Elles y campent «au bord du fleuve Amélès, dont aucun vase ne peut garder l'eau». Chaque âme doit boire de l'eau de ce fleuve. Celles qui n'ont pas assez de prudence boivent outre mesure. Puis elles oublient tout et elles s'endorment. A minuit, il survient un tremblement de terre et un éclat de tonnerre. En ce moment, toutes les âmes s'élancent vers les lieux où elles doivent renaître ${ }^{58}$. La reincarnation se présente ici comme l'éxigence de la Justice ${ }^{59}$, parce que la sorte de chaque vie nouvelle dépend du comportement de l'homme pendant sa vie passé $e^{60}$.

\section{LA POSSIBILITÉ D'EXERCER UNE INFLUENCE PAR LA VIE TERRESTRE SUR SA PROPRE DESTINÉE APRÈS LA MORT}

Nous avons déjà parlé de l'influence de l'existence de l'âme avant sa naissance sur sa vie terrestre et à la fois sur sa destinée après la mort. Maintenant nous voudrions considérer quelle est l'influence de la façon d'agir de l'homme dans sa vie sur sa destinée après la mort sous un aspect individuel, personnel.

Dans les Lois Platon dit que l'âme a sa place qui lui est donnée par le Dieu, selon ce qu'elle est devenue. Ce qu'elle deviendrait, le Dieu l'a fait dépendre d'elle-même. Il a laissé cela à notre volonté, à notre choix ${ }^{61}$. Cette liberté concerne le choix du genre de vie avant la naissance et aussi tous les choix que l'homme fait pendant la vie. Le Dieu fait passer vers une meilleure place

\footnotetext{
57 Cfr. Respublica 616 c-619 c ; Phaedo 81 d-82 b.

${ }^{58}$ Cfr. Respublica $620 \mathrm{~d}-621$ b.

59 Cfr. S. Lisiecki, Nauka Platona o prabycie duszy, Kraków 1927, 102.

${ }^{60}$ Dans le Phédon la théorie de l'incarnation est liée avec celle de la'rétribution, cfr. ibidem

61 Cfr. Leges 904 b.
} $72 \mathrm{~d}$ 
l'âme qui à formé en elle-même des meilleurs traits du caractère et vers une place pire celle qui a pris des traits mauvais ${ }^{62}$. C'est une exigence fondamentale de la justice.

Nous avons cité plus haut ce passage de Platon où il remarque qu'en chacun il existe deux motifs d'action que nous suivons. L'un, inné, c'est la recherche des plaisirs, l'autre, acquis, c'est l'aspiration vers le bien. Ces désirs sont en nous concordants ou non. Il arrive que ceci ou cela domine. Ce qui, par la raison, nous conduit au meilleur, c'est la tempérance ( $\sigma \omega \phi \varrho o \sigma u ́ v \eta)$. La supériorité du désir qui nous entraîne aux plaisirs et gouverne en nous, c'est la démesure (ưß@ı) $)^{63}$. C'est la même idée qui est exprimée dans les Lois. Platon dit:

«C'est dans cet universel dessein qu'il a imaginé [le Dieu - J.L.] en quelle place et en quels lieux il faut faire passer chaque être à mesure qu'il revêt telle ou telle qualité; quant à la production de ces qualités déterminées, il en a laissé les responsabilités aux volontés de chacun de nous. C'est, en effet, selon le sens de son désir et selon l'état de son âme, que, dans chaque occasion, chacun de nous prend, la plupart du temps, telle ou telle voie et telle ou telle qualitée ${ }^{64}$.

C'est le «dernier mot de l'eschatologie platonicienne», comme dit A. Diès $^{65}$. A.-J. Festugière a remarqué que nous avons ici deux principes: «le décret universel de la Providence et notre vouloir propre. Il n'y a point de marge, dans ce système, pour influence directe des planètes sur la conduite de l'homme» ${ }^{66}$. La sentence de Lachésis dans le mythe d'Er constate:

«La vertu n'a point de maître [...], la responsabilité est à qui a choisi et pas à Dieu» ${ }^{67}$.

C'est l'âme qui fait ses choix. C'est pourquoi les divinités de l'Olympe ont donné la sentence que «devenu plus méchant, on aille rejoindre des âmes plus méchantes; meilleur, des âmes meilleures; et que dans la vie comme dans toutes les morts successives, on subisse ou l'on exerce l'action naturelle du semblable sur le semblable» ${ }^{68}$. Or, les âmes qui n'ont pas subi beaucoup de mauvais changements et ceux qu'elles ont subis étaient légers et peu nombreux, se déplacent dans l'espace horizontalement. Si les changements sont plus profonds et les âmes ont fait beaucoup de mal, elles doivent aller aux endroits inférieurs et elles sont rongées par la souffrance pendant leur vie et après la

\footnotetext{
62 Cfr. ibidem 903 c.

63 Cfr. Phaedrus 237 d-238 a.

64 Leges 904 bc.

65 Cfr. A. Diès, in: Platon, Les Lois, livres VII-X, Paris 1956, p. 174.

66 A.J. Festugière, Platon et l'Orient, ,Revue de Philologie” 21 (1947) 34.

67 Respublica 617 e.

${ }^{68}$ Cfr. Leges 904 e.
} 
mort $^{69}$. Celles qui sont devenues meilleures et ont passé leur vie à la recherche du bien, vont aux lieux supérieurs, sous le ciel, où elles ont des temples et des bois où habitent des dieux avec lesquels elles demeurent face-à-face et ont beaucoup d'autres plaisirs vrais ${ }^{70}$.

L'âme qui se séparait de son corps pendant la vie, qui s'occupait de philosophie, va après la mort «vers ce qui lui ressemble, $[\ldots]$ vers ce qui est invisible, vers ce qui est divin et immortel et sage; c'est vers le lieu où son arrivée réalise pour elle le bonheur ", où tous les maux de la condition humaine cessent, où elle passe son temps dans la compagnie des dieux ${ }^{71}$. Les âmes des méchants doivent payer la peine de leur façon de vivre dans le corps:

«Elles errent jusqu'au moment où l'envie qu'en a leur acolythe, pour ce qui est de la corporéité, les fera de nouveau rentrer dans les liens d'un corps» ${ }^{72}$.

En disant plus concrètement: dans les formes d'ânes ou d'autres bêtes semblables vont les âmes de ceux qui avaient péché par gloutonnerie, impudicité, beuverie. Dans des formes de loups, de faucons, de milans existent celles, qui pendant leur vie, avaient péché par injustice, tyrannie, rapine. Au contraire, ceux qui s'occupaient d'exercer une activité sociale et civique pour lesquelles ils devaient avoir de la vertu, qui se nomme tempérance et justice, vont dans les corps des animaux qui sont sociables et policé: abeilles, guêpes, fourmis ou bien des hommes ${ }^{73}$. Une position tout exceptionnelle est conférée à ces âmes qui ont appartenu aux philosophes. Elles montent parmi les dieux où elles ont leurs résidences ${ }^{74}$.

Nous avons présenté les images mythologiques de l'eschatologie platonicienne et aussi à un certain point le contenu d'enseignement qu'elles renferment. On doit enfin considérer quelle est la relation entre le mythe ( $\mu \tilde{v} \theta$ os) et

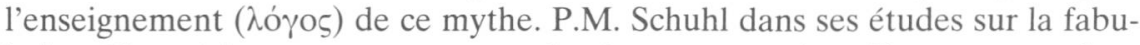
lation Platonicienne remarque que les images ont chez Platon une valeur symbolique. Les mythes ne nous donnent pas les images complètes et exactes. Leur valeur se fonde aussi sur ce qui ne peut pas être exprimé, qui se trouve au fond $d^{75}$. On peut dire que le mythe est une image fausse, mais c'est l'image de

${ }^{69}$ Cfr. ibidem 904 c-d.

${ }^{70}$ Cfr. ibidem 111 b-c.

71 Cfr. Phaedo 81 a.

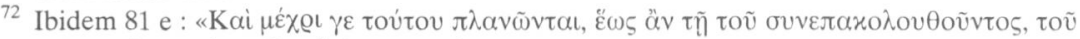

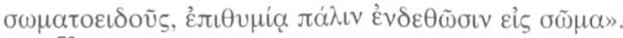

${ }^{73}$ Cfr. ibidem 82 a-b.

${ }^{74} \mathrm{Cfr}$. ibidem $82 \mathrm{~cd}$ et $69 \mathrm{c}$. 
la vérité. «Le mythe n'est autre chose qu'une fiction qui représente la vérité» comme dit Olympiodore ${ }^{76}$.

Les images que nous donne Platon dans le Gorgias, Phédon La République, Phèdre sont de plus en plus riches. Elles sont comme les divers vêtements, qui procèdent de la tradition orphique et pythagoricienne ou de termes scientifiques $^{77}$. Elles lient l'eschatologie avec la physique. Les mythes-images n'illustrent pas seulement le thème mais le transforment (refont) ${ }^{78}$. La fonction propre du mythe c'est d'exprimer «en termes concrets les raisonnements abstraits inaccessibles au vulgaire». Les mythes, comme remarque Schuhl, «traduisent ce qui en peut être transposé en images sensibles, indiquent une orientation, une direction» ${ }^{79}$. Les mythes sont «certaines représentations sensibles, ou certains appareils [...] qui permettent de faire comprendre au vulgaire même des relations abstraites, mais non les plus hautes, que le raisonnement seul peut faire connaître» ${ }^{80}$. Le mythe n'est pas seulement transposition vulgarisatrice ou bien exposition scientifique. "Il est aussi [...] incarnation; et il insère la destinée de l'âme dans un ordre cosmique, lui-même animé»" ${ }^{81}$. "Or les mythes ont été inventés pour que nous allions de ce qui est apparent à ce qui est invisible»- comme nous assure Olympiodore ${ }^{82}$. Avant tout c'est très interessant de suivre l'influence de l'eschatologie platonicienne sur la chrétienne en voyant quand elles vont en accord et quand elles diffèrent.

\section{MIT ESCHATOLOGICZNY U PLATONA \\ Wprowadzenie}

(Streszczenie)

Artykuł w swej treści jest przypomnieniem głęboko zakorzenionych w kulturze Zachodu idei dotyczących eschatologii, które zostały wprowadzone do tradycji myśli europejskiej poprzez twórczość Platona. Obejmują one problemy związane z sądem

75 Cfr. P.M. Schuhl, La fabulation Platonicienne, Paris 1947, 64.

76 Olympiodorus, In Platonis Gorgiam commentaria 46, 3, ed. L.G. Westerink, Leipzig 1970, trad. V. Cousin: Fragments philosophiques, in: Philosophie ancienne, Paris 1840², 389: «Mṽos

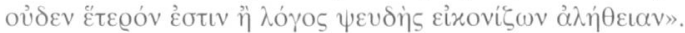

77 Cfr. Schuhl, La fabulation Platonicienne, p. 56.

78 Cfr. ibidem, p. 57.

79 Ibidem, p. 21.

80 Ibidem, p. 37

81 Ibidem, p. 22

${ }^{82}$ Cfr. Olympiodorus, In Platonis Gorgiam commentaria 46, 2: «Kai oi $\mu \tilde{v} \theta$ ol toívuv

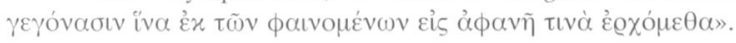


pośmiertnym i retrybucją za czyny dokonane za życia, z reinkarnacją i preegzystencją. Uwzględniając główne idee antropologiczne zawarte w pismach Platona autor przedstawia te treści, które odnoszą się w nich do celu życia ludzkiego i losów człowieka po śmierci. W ramach tych ostatnich omawia zagadnienia związane z sądem - sędziowie i miejsce sądu, wyrok i miejsce przeznaczenia: Tartar i niebo, następnie zasadę retrybucji, wielkość i czas trwania kary, reinkarnację i powrót do nowego życia na ziemi, możliwość wpływania przez swoje postępowanie za życia na swe przeznaczenie po śmierci. Wreszcie po omówieniu obrazów mitologicznych została przedstawiona zasada oceny ich przydatności dla ukazania eschatologii człowieka. Opiera się ona na uwzględnieniu relacji między literackim mitem ( $\mu$ ṽ 0 s) a wypływającą z niego nauką ( $\lambda$ ó $\gamma$ os). Istotną dla zrozumienia tej relacji jest świadomość, że mit ma wymiar symboliczny, ponieważ nie może wyrazić całej prawdy. Zgodnie z określeniem podanym przez Apollodora jest on opowiadaniem zmyślonym obrazującym prawdę. Jako takie służy możliwie szerokiemu ukazaniu odbiorcy o prostym umyśle tego, do czego filozof dochodzi w abstrakcyjnym rozumowaniu, a tym samym pozwala przejść od tego, co jest w punkcie wyjścia obrazem zmysłowym do rzeczywistości niepostrzegalnej zmysłami. 